\title{
Identificação de enfermidades agudas causadas por animais e plantas em ambientes rurais e litorâneos: auxílio à prática dermatológica
}

\author{
Identification of acute diseases caused by animals and plants in wild \\ environments: contribution to dermatologic practice
}

\begin{abstract}
Vidal Haddad Junior ${ }^{1}$
Resumo: Fundamentos: Nos últimos anos, existe um aumento progressivo do contato de moradores urbanos com ambientes selvagens devido a atividades de lazer. Com isso, algumas dermatites pouco conhecidas podem ser observadas nas clínicas privadas e ambulatórios dermatológicos, especialmente nos inícios de semana e finais de férias.

Овлетіvos: Obter e fornecer informações para dermatologistas sobre o problema.

PaCientes e Métodos: O autor observou, em adultos e crianças, dermatites agudas associadas a plantas ou animais em Ubatuba, cidade litorânea de São Paulo, por dois meses (junho/julho de 2006) e na Faculdade de Medicina de Botucatu, também por dois meses (junho/julho de 2007).

RESUltados: Foram observados 25 pacientes na área rural e 43 na área litorânea nas condições estabelecidas. Em áreas rurais, foram mais observadas fitofotodermatites e picadas de insetos, enquanto em áreas litorâneas traumas por ouriços-do-mar e fitofotodermatites predominaram; entretanto, em ambas as áreas ocorreram outros acidentes de difícil identificação na prática diária.

ConclusóEs: Devemos estar atentos ao fato de o paciente procurar o dermatologista somente após as fases agudas dos acidentes. Informações sobre as enfermidades mais comuns e suas características podem ser muito úteis para a prática nos consultórios. O autor sugere uma tabela algorítmica para auxílio diagnóstico.
\end{abstract}

Palavras-chave: Animais peçonhentos; Dermatologia; Exposição ambiental; Intoxicação por plantas; Intoxicação por plantas/etiologia

\begin{abstract}
BACKGROUND: In recent years, there has been increasing contact between human beings that live in urban regions and the wild environment due to a series of activities. As a result, some poorly known dermatitis may present in private and dermatological clinics, especially early in the week and at the end of vacation periods.

OBJECTIVES: To obtain and provide information for dermatologists on the problem.

PATIENTS AND Methods: The author observed adult and pediatric patients with acute dermatitis associated with plants or animals in Ubatuba, coastal city of Sao Paulo for 2 months (June / July 2006) and Medical School of Botucatu (June / July 2007).

RESULTS: We observed 25 patients in the rural area and 43 in the coastal environment. In rural areas, the most common dermatitis were phytophotodermatitis and the stings of insects and in coastal areas, sea urchin injuries and phytophotodermatitis, but in both areas there were some other accidents that were difficult to identify by regular daily practice.

Conclusion: We should be alert to the fact that patients seek a dermatologist after the acute phase of injuries. Information on the most common diseases and their characteristics can be very useful to practice in dermatologic clinics. The author suggests an algorithm to support diagnosis.

Keywords: Animals, poisonous; Dermatology; Environmental exposure; Plants poisoning;

Plants poisoning/etiology
\end{abstract}

Recebido em 26.04.2009.

Aprovado pelo Conselho Consultivo e aceito para publicação em 29.05.09.

* Trabalho realizado na Santa Casa de Misericórdia de Ubatuba - Ubatuba (SP), Brasil - e na Faculdade de Medicina de Botucatu - Universidade Estadual Paulista (Unesp) - Botucatu (SP), Brasil.

Conflito de interesse: Nenhum / Conflict of interest: None

Suporte financeiro: Nenhum / Financial funding: None

Professor doutor da Faculdade de Medicina de Botucatu (Universidade Estadual Paulista) - Botucatu (SP), Brasil - e médico colaborador do Hospital Vital Brazil, Instituto Butantan - São Paulo (SP), Brasil.

(C) 2009 by Anais Brasileiros de Dermatologia 


\section{INTRODUÇÃO}

Embora algumas dermatites causadas por plantas e animais possam ocorrer em ambientes urbanos, a maioria dos casos acontece após contato com ambientes rurais. ${ }^{1,2,3}$ Atualmente, vivemos uma intensa valorização de atividades esportivas e de lazer, o que direciona o ser humano para esses locais. Com isso, contatos com vários animais e plantas podem ocasionar dermatites polimorfas de frequência e gravidade variáveis. Elas são, na maioria das vezes, causadas pelo contato direto com o agente e associadas a toxinas ou fenômenos alérgicos, diferindo das enfermidades comumente observadas nos consultórios. Os acidentes mais comuns causados por animais peçonhentos são provocados por artrópodes, como os besouros (especialmente dos gêneros Paederus e Epicauta), abelhas, aranhas (gêneros Loxosceles e Pboneutria) e formigas (particularmente as formigas lava-pés, do gênero Solenopsis). As aroeiras e plantas fotossensibilizantes são responsáveis pela maioria dos agravos causados por plantas. O conhecimento dos agravos mais comuns e o relato da estadia recente em ambiente aberto são extremamente úteis. Deve-se ainda ter em mente que o paciente será examinado pelo dermatologista apenas no retorno ao ambiente urbano, uma vez que o primeiro exame é realizado na maioria das vezes por um profissional que atua em pronto-socorro no local onde ocorre o acidente.

\section{OBJETIVOS}

Fornecer dados para identificação dos agentes causadores e alertar os dermatologistas para a possibilidade do atendimento de enfermidades provocadas por plantas e animais de locais silvestres em seus locais de prática diária.

\section{CASUÍSTICA E MÉTODOS}

$\mathrm{O}$ autor registrou dermatites causadas por animais e plantas em 68 adultos e crianças durante um período de dois meses (junho e julho), a partir de observações dirigidas em atendimentos realizados na Santa Casa de Ubatuba, litoral norte do Estado de São Paulo (junho/julho de 2006), e no ambulatório e triagem da Faculdade de Medicina de Botucatu, Universidade Estadual Paulista (junho/julho de 2007). As observações iniciais dos pacientes foram efetuadas pelo corpo clínico das instituições, em regime de rotina. A orientação do autor se fez após pré-seleção dos acidentados pelas equipes. Os pacientes cujo agravo tinha etiologia duvidosa quando do exame dermatológico e aqueles que não puderam dar informações com clareza foram excluídos do estudo. Os pacientes foram convidados a responder a um questionário dirigido e autorizaram o uso dos dados para uma análise descritiva dos quadros por meio de um protocolo assinado.

\section{RESULTADOS}

Foram observados 25 pacientes na área rural (Botucatu) e 43 na área litorânea (Ubatuba) nas condições exigidas pelo estudo. Aproximadamente 50\% (33) eram crianças (menores de 12 anos). Todos os pacientes apresentaram comprometimento cutâneo em maior ou menor grau, e cerca de $70 \%$ manifestaram intenção de procurar um dermatologista quando retornassem à cidade de origem. Os pacientes foram classificados pelos tipos de lesões que apresentaram e estas foram associadas aos agentes (Tabelas 1, 2 e 3). Apenas sete pacientes (cerca de 10\%) trouxeram os agentes causadores, e nenhum trouxe plantas, apesar de haver associação posterior das lesões com a proximidade destas.

\section{DISCUSSÃO}

O município de Botucatu apresenta florestas, rios e montanhas, onde é possível fazer caminhadas e praticar a pesca, o ciclismo e outros esportes. As fitofotodermatites foram comuns, devido à manipulação

TABELA 1: Algoritmo para identificação de acidentes causados por animais e plantas quando das manifestações agudas

Tipos de Lesões

Máculas, placas e vesículas lineares, de distribuição bizarra

Nódulos inflamatórios com orifício central

Nódulos eritematosos com espículas no local

Pápulas e placas inflamatórias (dor residual)

Seropápulas pruriginosas

Pustulose (membros inferiores)

Quadros eczematosos extensos

Necrose cutânea de vários graus (membros inferiores)

Vesículas e bolhas em áreas expostas (região cervical)
A - Fitofotodermatite, cnidários

B - Miíase furunculoide

C - Ouriços-do-mar

D - Abelhas, lagartas, aranhas

E - Carrapatos, prurido agudo

F - Formigas

G - Plantas sensibilizantes (aroeira)

H - Serpentes (Bothrops), peixes peçonhentos (bagres)

I - Besouro urticante 
TABela 2: Dermatites agudas em área rural montanhosa e florestal

\begin{tabular}{lcc}
\hline Lesão & Casos & Tipo \\
\hline Fitofotodermatite & 7 & A \\
Prurido agudo & 5 & E \\
Abelhas & 3 & D \\
Carrapatos & 3 & E \\
Miíase & 2 & B \\
Aranhas & 1 & D \\
Besouro urticante (potó) & 1 & I \\
Formigas & 1 & F \\
Lagarta & 1 & D \\
Plantas sensibilizantes (aroeira) & 1 & G \\
\hline
\end{tabular}

TABELA 3: Dermatites agudas em área litorânea

\begin{tabular}{lcc} 
Lesão & Casos & Tipo \\
\hline Ouriços-do-mar & 14 & $\mathrm{C}$ \\
Prurigo agudo (mosquitos) & 12 & $\mathrm{E}$ \\
Fitofotodermatite & 6 & $\mathrm{~A}$ \\
Cnidários (águas-vivas) & 4 & $\mathrm{~A}$ \\
Peixes peçonhentos (bagres) & 3 & $\mathrm{H}$ \\
Abelhas & 2 & $\mathrm{D}$ \\
Serpentes (Bothrops) & 1 & $\mathrm{H}$ \\
Carrapatos & 1 & $\mathrm{E}$ \\
\hline
\end{tabular}

de frutas cítricas ${ }^{4}$ (Figura 1). As picadas de moscas e mosquitos vieram a seguir, associadas ou não a fenômenos alérgicos ${ }^{5}$ (Figura 2), assim como as picadas únicas de abelhas. ${ }^{2}$ Nos ambientes rurais, em determinadas épocas, ocorre parasitismo por carrapatos, especialmente pelos gêneros Ixodes e Amblyomma, causadores de uma dermatite vesiculosa extremamente pruriginosa. Esses artrópodes são incriminados ainda por transmitir doenças como a febre maculosa e a doença de Lyme (Figura 2). Dor intensa, pápulas e/ou placas se associam a acidentes por animais peçonhentos, como as lagartas, ${ }^{2,6}$ sendo as da família Megalopigidae as mais comuns. Já pustuloses súbitas em crianças com história de estadia no campo podem ser causadas por formigas lava-pés (Solenopsis invicta), embora a fase logo após a picada (antes de 24 horas) mostre pápulas eritemato-edematosas. ${ }^{2,7}$ Vesículas e bolhas nas áreas expostas (especialmente na região cervical) podem ser causadas por besouros urticantes (potós, pertencentes ao gênero Paederus), ${ }^{2}$ animais comuns especialmente nas regióes Norte, Nordeste e Centro-Oeste, mas presentes em todo o país (Figura $3)$. Necroses cutâneas podem ser provocadas por peixes peçonhentos (arraias e bagres fluviais, por exemplo) ou por serpentes do gênero Bothrops ${ }^{1,2}$ - as jararacas, urutus e jararacuçus (Figura 4). Quadros eczematosos, generalizados e agudos, são sugestivos de
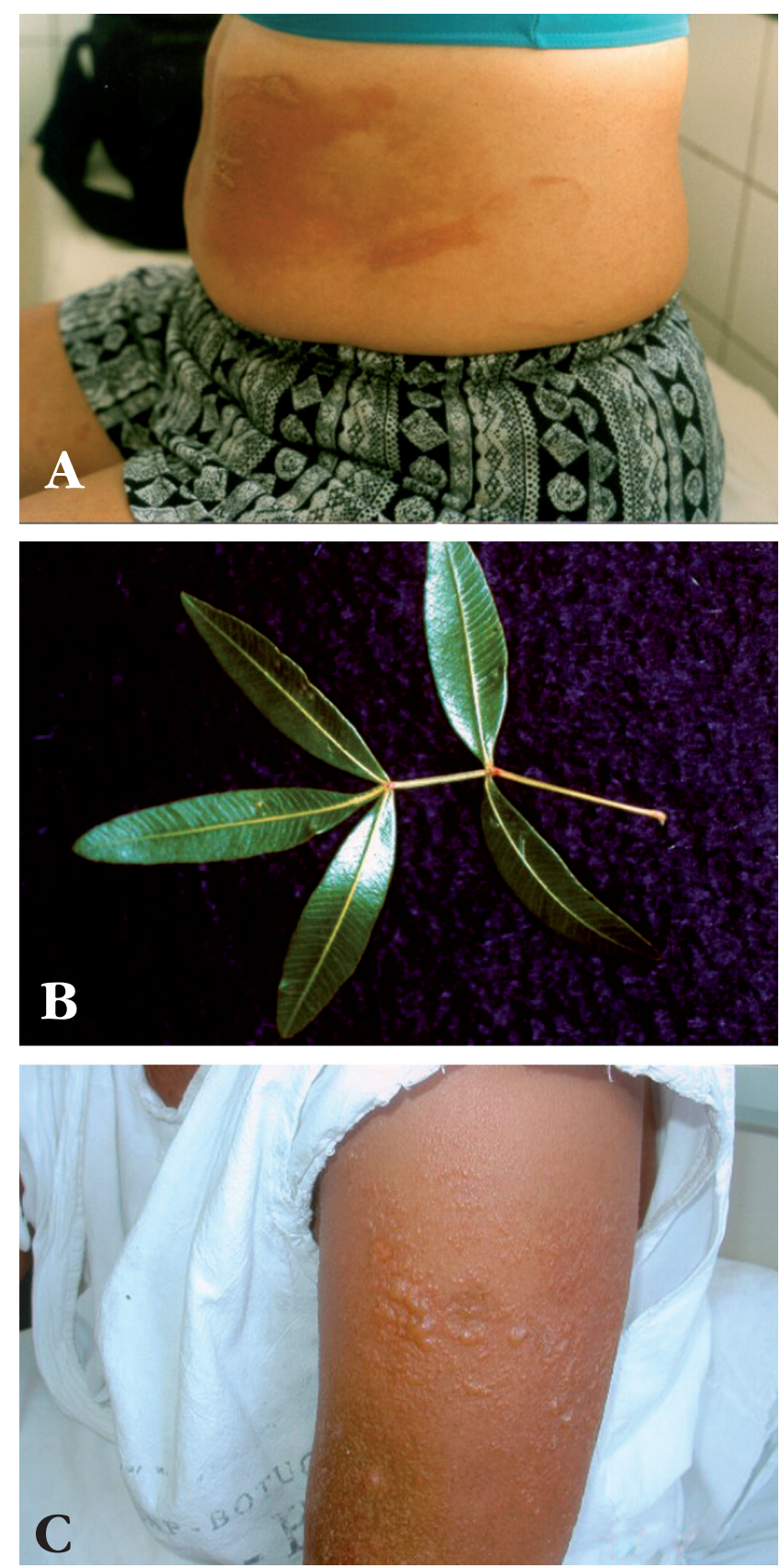

Figura 1: A. Fitofotodermatite típica, ocorrendo marcas bizarras do contato com os dedos da paciente após manipulação de limão; B. Folha de aroeira; C. Dermatite aguda no braço da paciente causada pela aroeira

contato com os fenóis dispersos no ar por plantas da família Anacardiaceae, especialmente as $\operatorname{aroeiras}^{4}$ (Figura 1).

Em ambientes litorâneos, como os observados em Ubatuba, existe o risco de acidentes semelhantes aos das áreas rurais, pelo contato crescente com as matas e cursos fluviais, mas a maioria dos acidentes está associada ao mar. ${ }^{1,8}$ Novamente encontramos uma grande incidência de fitofotodermatites, pelo advento 

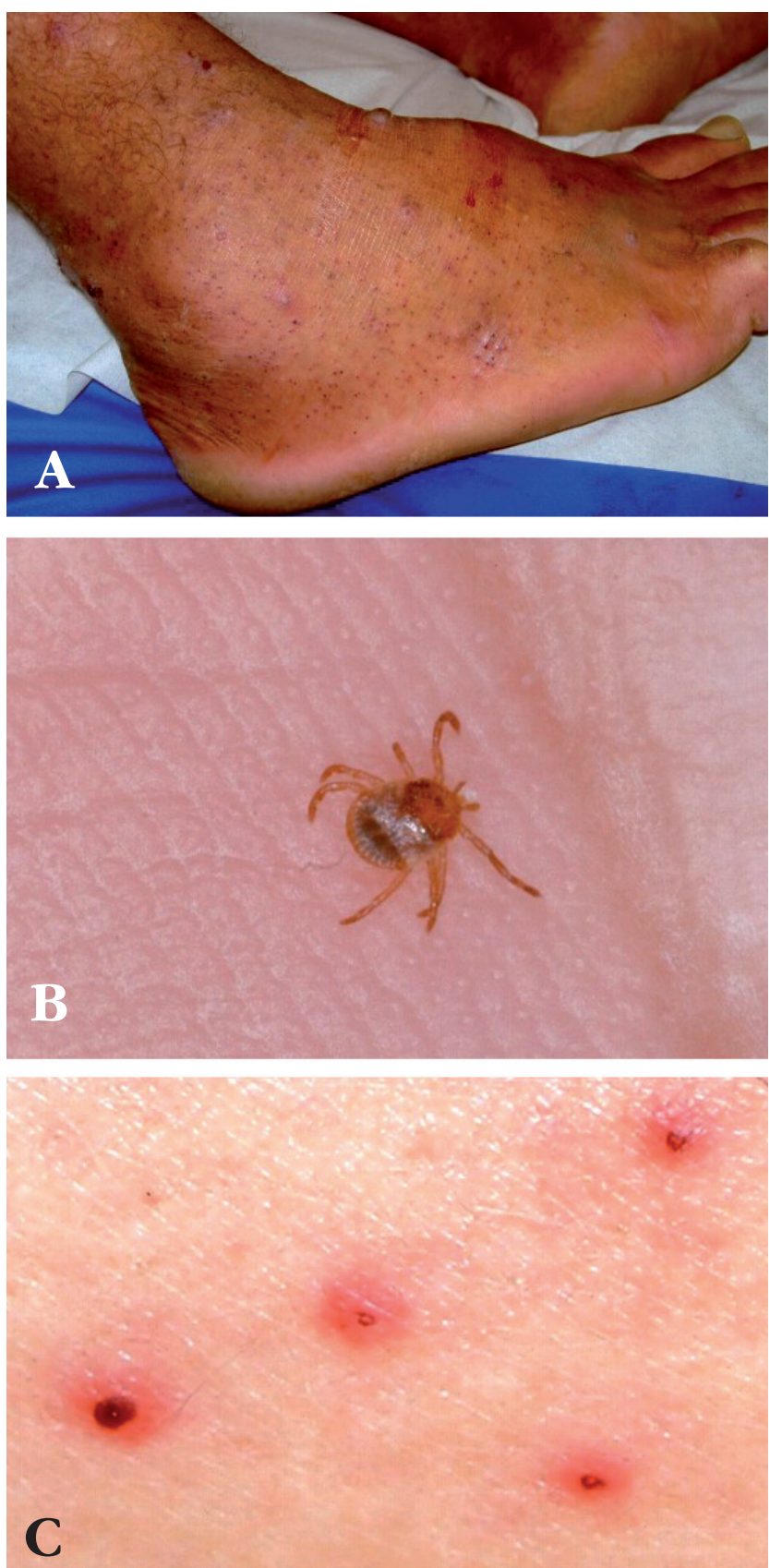

Figura 2: A. Picadas de borrachudos ou piuns (gênero Simulium). Em regiões praianas ocorrem ataques maciços contra pessoas; $\mathrm{B}$. Carrapato do gênero Ixodes; C. Picadas de carrapato. Notar as pápulas eritematosas e as exulcerações centrais resultantes do rompimento de vesículas

do turismo, mas os problemas mais comuns foram acidentes por ouriços-do-mar. Pisados pelos banhistas, eles causam inflamação inicial seguida de uma dermatite nodular tardia com presença de espículas, geralmente nas regiões plantares ${ }^{1,2,9}$ (Figura 5). Os acidentes por águas-vivas e caravelas (cnidários) também são comuns e assemelham-se aos quadros de fitofotodermatites, podendo haver confusão diagnóstica, o que será mais provável na fase não inflamatória inicial
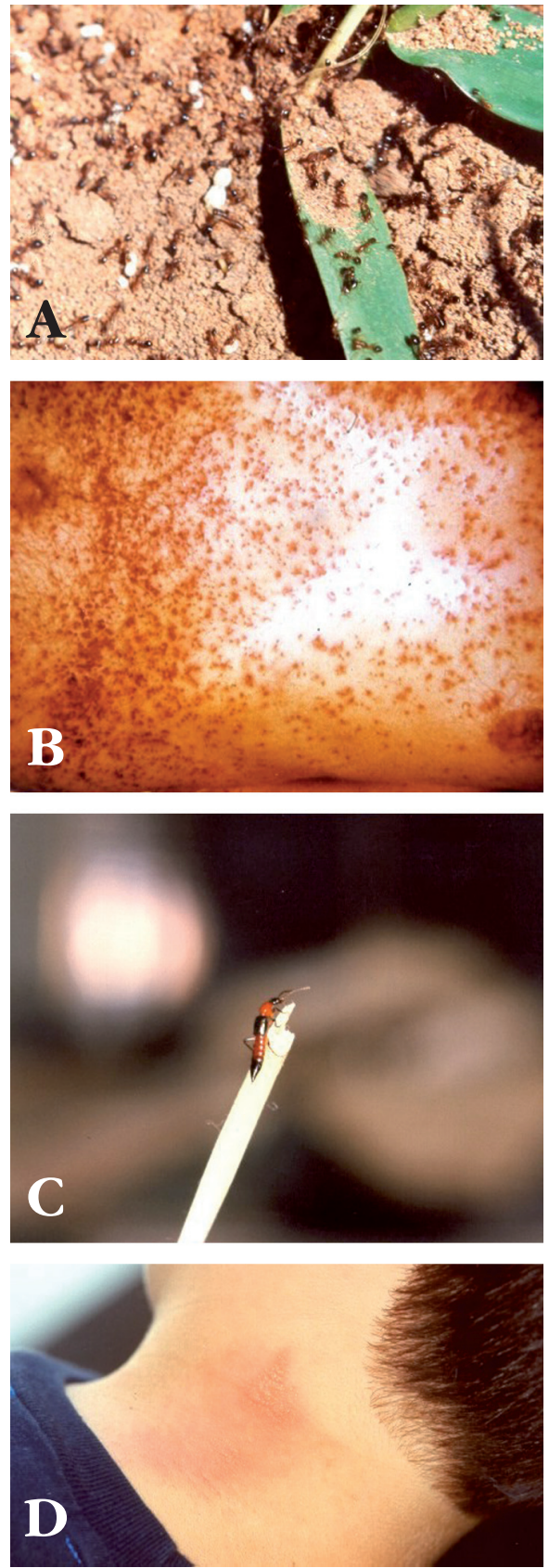

Figura 3: A. Formigas lava-pés (Solenopsis invicta); B. Quadro pustuloso extenso causado por formigas lava-pés; C. Besouro urticante (potó, gênero Paederus); D. Vesículas confluentes em área típica em um paciente, causadas pelo potó

(cerca de 24 horas após o contato) ${ }^{1,210,11}$ (Figura 6).

O tratamento dos acidentes varia de acordo com a etiologia: ${ }^{1,2}$ acidentes com inoculação de peçonhas devem primariamente ser tratados com soro antiveneno, mas isso só é possível em envenenamentos por serpentes e aranhas (todos os envenenamentos por serpentes e alguns por aranhas ${ }^{2}$ ), uma vez que não são produzidos soros contra o veneno da maioria dos animais peçonhentos. Acidentes por lagartas, cnidários e 

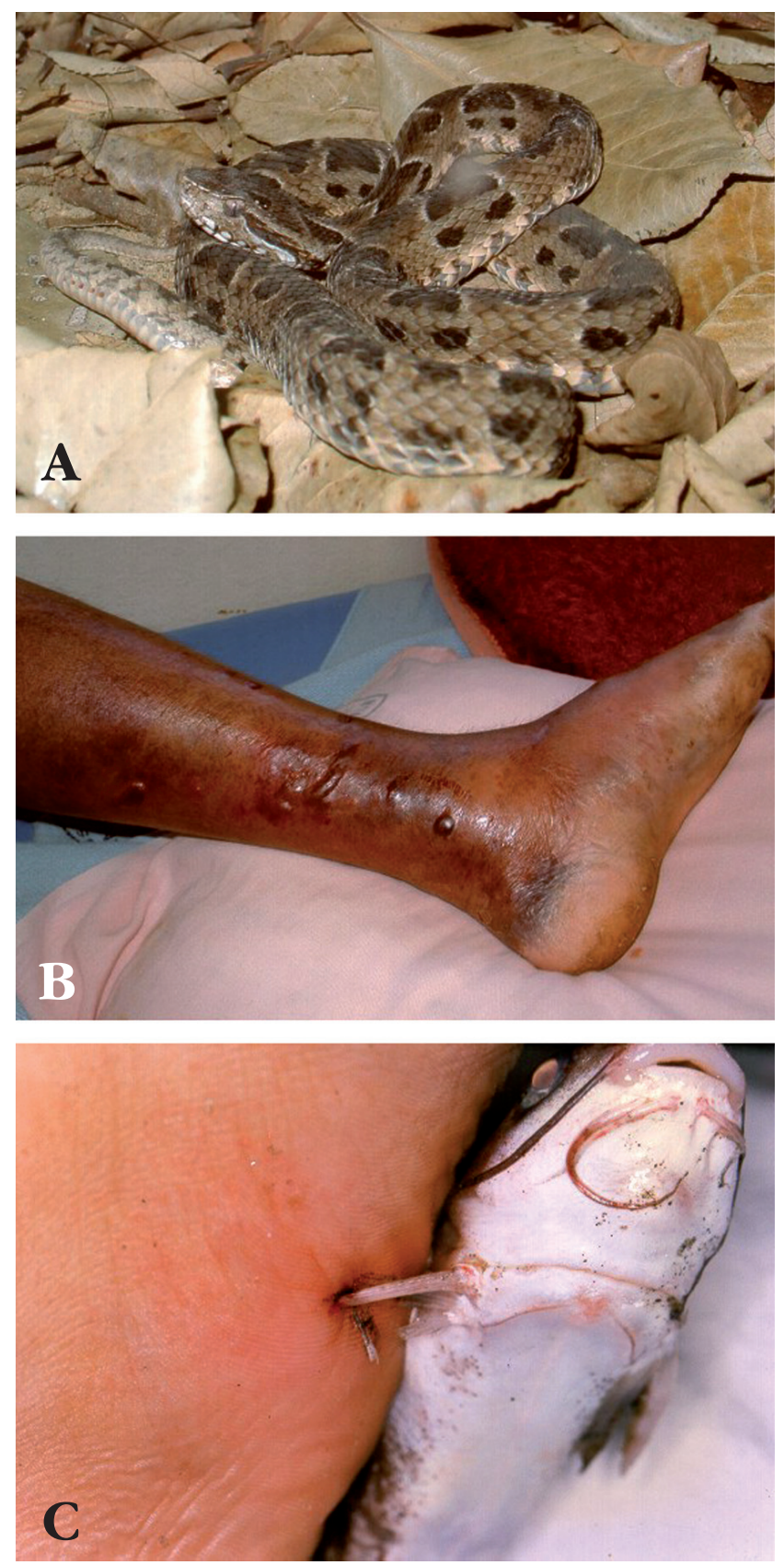

Figura 4: A. Serpente do gênero Bothrops; B. Acidente por Bothrops (necrose e sangramento); C. Ferimento causado por um bagre (Genidens genidens) em praia de Ubatuba (SP)

peixes peçonhentos só podem ser medicados sintomaticamente: no caso das lagartas, com o uso de analgésicos e, por vezes, anestesia troncular; para os cnidários, com o uso de água do mar gelada e compressas de vinagre; e para os peixes peçonhentos, com imersão em água quente. Os corticoides tópicos podem ser empregados nas dermatites irritativas por contato como a fitofotodermatite -, nas dermatites por besouros, nos quadros eczematosos por plantas, no prurido
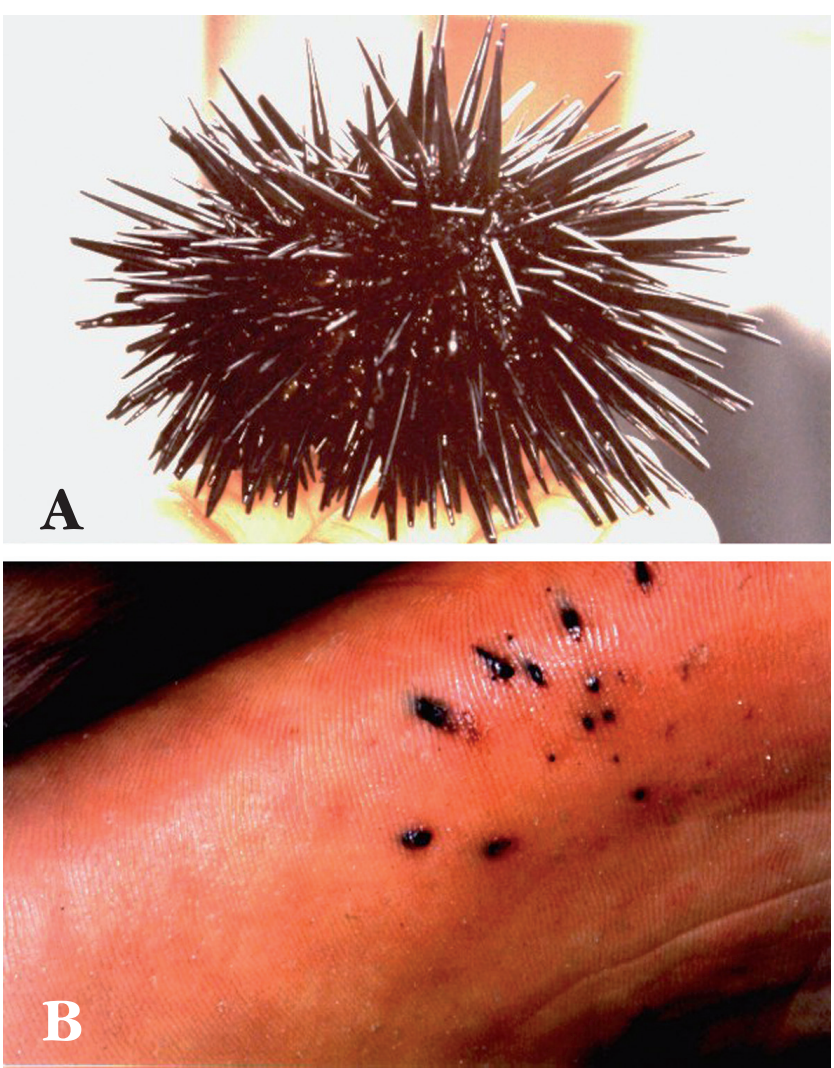

Figura 5: A. Ouriço-do-mar; B. As espículas negras confirmam o diagnóstico de acidente por ouriço-do-mar

agudo e nas picadas de carrapato. Miíases devem ser extraídas por expressão ou com o auxílio de pinças, assim como as espículas de ouriços-do-mar. ${ }^{1-7}$

\section{CONCLUSÕES}

Existem diferenças entre as fases agudas de acidentes por animais e plantas e as manifestações apresentadas pelos pacientes após 24 a 48 horas. Inicialmente, é mais provável que todas as dermatites aqui apresentadas serão observadas por profissionais no setor de emergência dos hospitais, mas em um ou dois dias a probabilidade de um dermatologista ser procurado aumenta, em razão de a totalidade dos pacientes apresentarem manifestações cutâneas. A dor, elemento importante logo após o contato com animais peçonhentos, como lagartas, cnidários, serpentes e peixes, não estará presente quando o paciente for examinado em um consultório. Da mesma forma, lesões cutâneas podem mudar sua apresentação, como ocorre nas picadas de formigas lava-pés, que formam pústulas sobre as pápulas urticadas iniciais em cerca de 24 horas. Fitofoto- 

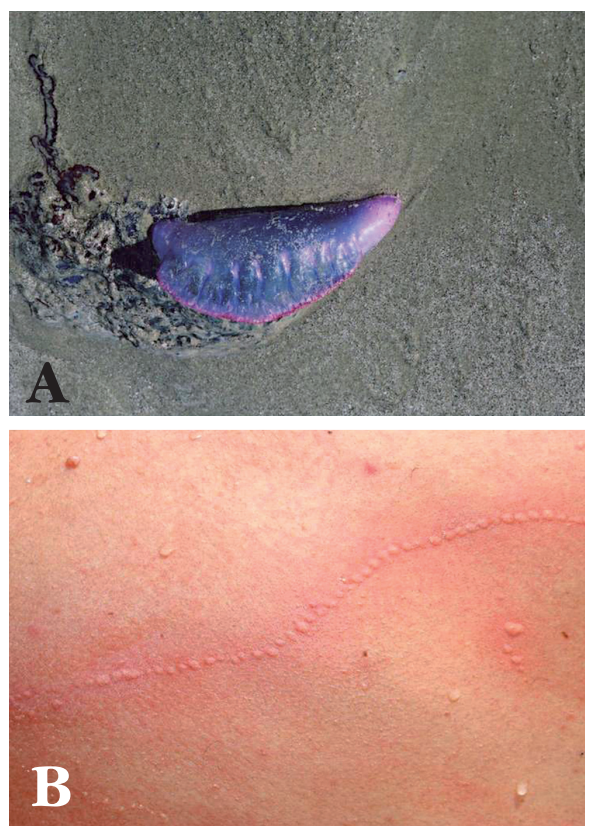

Figura 6:

A. Caravela

(Pbysalia

pbysalis);

B. Acidente típico

linear. A dor

imediata é

importante na

diferenciação com

fitofotodermatites

\section{REFERÊNCIAS}

1. Haddad Jr V. Animais aquáticos potencialmente perigosos do Brasil: guia médico e biológico. São Paulo: Editora Roca; 2008.

2. Cardoso JLC, França FOS, Wen FH, Malaque CMS, Haddad Jr V. Animais Peçonhentos no Brasil: biologia, clínica e terapêutica dos acidentes. São Paulo: Editora Sarvier; 2003.

3. Haddad Jr V. Infecções cutâneas e acidentes por animais traumatizantes e venenosos ocorridos em aquários comerciais e domésticos no Brasil: descrição de 18 casos e revisão do tema. An Bras Dermatol. 2004; 79:157-67.

4. Haddad Jr V. Skin manifestations caused by Brazilian traumatic, allergenic and venomous plants: main species, therapeutic and preventive measures. J Venom Anim Toxins incl. Trop Dis. 2004;10: 199-206.

5. Miot HA, Baptistella RF, Batista KA, Volpato DEC, Augusto LST, Madeira NG, et al. Comparative study of the topical efectiveness of the Andiroba oil (Carapa guianensis) and DEET 50 percent as repellent for Aedes sp. Rev Inst Med Trop Sao Paulo. 2004;46:253-6.

6. Cardoso AEC, Haddad Jr V. Acidentes por Lepidópteros (larvas e adultos de mariposas): estudo dos aspectos epidemiológicos, clínicos e terapêuticos. An Bras Dermatol. 2005;80:571-8.

7. Haddad Jr V, Cardoso JLC, França FOS, Wen FH. Acidentes por formigas: um problema dermatológico. An Bras Dermatol. 1996;71:527-30.

dermatites, que geram pouca sintomatologia, podem tornar-se extremamente semelhantes a acidentes com águas-vivas e caravelas após 24 a 48 horas, quando estes já não apresentam sua dor característica. O conhecimento sobre as enfermidades mais comuns e suas características pode ser muito útil para a prática nos consultórios e ambulatórios. Para isso, é fornecida uma tabela algorítmica (Tabela 1) que mostra os principais sinais e sintomas presentes em pacientes acidentados por plantas e animais silvestres (incluindo os peçonhentos) 24 a 48 horas após os acidentes, enfatizando a importância do exame clínico e da identificação das alterações ocorridas desde o contato inicial em áreas silvestres.

8. Haddad Jr V. Animais aquáticos de importância médica. Rev Soc Bras Med Trop. 2003;36:591-7.

9. Haddad Jr V, Novaes SPMS, Miot HA, Zuccon A. Accidents caused by sea urchins - the efficacy of precocious removal of the spines in the prevention of complications. An Bras Dermatol. 2001;76:677-1681.

10. Haddad Jr V, da Silveira FL, Cardoso JLC, Morandini AC. A report of 49 cases of cnidarian envenoming from southeastern Brazilian coastal waters. Toxicon. 2002; $40: 1445-50$.

11. Haddad Jr V, França FOS, Wen FH, Cardoso JLC. Acidentes provocados por celenterados: aspectos clínicos e terapêuticos. An Bras Dermatol. 1997;72:206-10.

ENDEREÇO PARA CORRESPONDÊNCIA / MAILING ADDRESS:
Vidal Haddad Junior
Caixa POStal 557
18618 OOO Botucatu SP
Tel./Fax: (14) 3882-4922
baddadjr@fmb.unesp.br

ENDEREÇO PARA CORRESPONDÊNCIA / MAILING ADDRESS:

Vidal Haddad Junior

Caixa Postal 557

18618000 Botucatu SP

baddadjr@fmb.unesp.br

Como citar este artigo/How to cite this article: Haddad Jr V. Identificação de enfermidades agudas causadas por animais e plantas em ambientes rurais e litorâneos: auxílio à prática dermatológica. An Bras Dermatol. 2009;84(4):343-8. 